\title{
Stress Distribution in Specimen for Diametrical Compression Test under Some Boundary Conditions
}

\author{
Shinnosuke Yoshinaga ${ }^{1}$, Yukari Higashi ${ }^{1}$, Rini Asnida Abdullah ${ }^{2}$ \& Takashi Tsutsumi $^{1 *}$ \\ ${ }^{1}$ Advanced course of Civil Engineering, National Institute of Technology, Kagoshima College, \\ Kirishima, Kagoshima, 899-5193, Japan \\ ${ }^{2}$ Department of Civil Engineering, Universiti Teknologi Malaysia, 81310 Johor Bahru, Malaysia
}

\begin{abstract}
Diametrical Compression test is one of indirect tests to investigate the tensile strength of brittle materials. This test is spread because of easiness to perform. However, results from this test are not stable. Traditionally, calculations for diametrical compression test are performed under a pair of opposite concentrated load on the diameter of specimen. In the fact, contact areas are appears just before fracture of specimen and the stress distribution from loading platens is not obvious. It seems one of reasons why the result from this test is not stable. Several stress distributions between contact area and specimen have been proposed and stresses and strains have been calculated as theoretical results or analytical result in previous studies. In this study, the stress distribution that consists of uniform loading and cosine curve shaped loading is proposed and used for theoretical solution. Furthermore, results from this study are compared with results from previous studies.
\end{abstract}

\section{Introduction}

Diametrical compression test (also termed as indirect tensile strength test) is a method for measuring tensile strength of a brittle material like rocks. The test is performed by placing a disc-shaped sample (disc thickness twice its diameter) in between two rigid platens. Compressive load is applied via steel platen which is in contact with the sample. Since tensile strength of brittle material is much smaller than its compressive strength. Therefore, the brittle material fails under tensile manner, usually in the form of vertical diametrical fracture.

Direct tensile test is another method to investgate the strength of britte materials. The accuracy of result from this test is higher than that from diametrical compression test. However, there are some difficulities to prepare specimens for this test. Furthermore, any eccentricity generating at the both edge of specimen has to be avoided when this test is carried out. In addition, equipments for direct tensile test are expensive. Therefore, diametrical compression test is spread especially in rising nations.

One of weak points in diametrical compression test is instability of its results. Traditionally, calculations for diametrical loading test is performed under a pair of opposite concentrated load on the diameter of specimen. From this assumption, for an isotropic material, the 
tensile stress reaches a maximum at a constant magnitude of $P /(\pi a)$, where $P$ represents the applied load and $a$ represents the radius of the circular plane [1]. However, contact areas appear just before fracture of specimen in the fact and the stress distribution from loading platens is not obvious. It seems one of reasons why the result from this test is not stable.

Several attempts have been seen in modeling for boundary condition. Cauweleart et al [2] obtained a solution using the theoretical results for a semi-infinite plate under concentrated force. The theoretical solution for orthotropic specimen under concentrated force was shown by Claesson and Bohloli[3], Exadaktylos [4] and Exadaktylos and Kaklis[5]. The theoretical solution under uniform radial load distributed on whole contact area was shown and compared with the result from some experiments by Chen et al [6] and Markides et al.[7]. Analytical models using finite element were used by Lemmon and Blackketter [8] to evaluate the distribution of tensile stress for diametrical compression test. The contact area between the specimens and two loading platens was set at 2, 4 and $8 \%$ of the circumference of the specimen with uniform loading whose direction is same as loading fromplatens. The results of this study were in agreement with the theoretical model, using orthotropic stress functions under the same conditions, as proposed by one of authors [9]. The results show that the tensile stress generating near the loading platens is influenced by the contact area between the loading platens and the specimen. For isotropic materials (i.e., with material properties that are uniform along each axis), the maximum tensile stress generates at the center of disk when the contact area increases. For orthotropic materials, the maximum tensile stress also generates at the center of circular plane when the angle of the principal material direction to the loading direction is close to $\pi / 4$. And the maximum value is almost constant regardless of the contact area. On the other hand, the maximum tensile stress generates near the loading platen decreases with increasing contact area, when the angle between the principal material direction and the loading direction is small. The latter shows that that contact area has a significant influence on tensile strength measurements.

Therefore, especially for orthotropic materials, it should be required to define the distribution of loading on the contact area to improve the accuracy for measurement of tensile strength using diametrical compression test. In previous studies, the distribution of stresses applied on the loading platens was assumed to be uniform. In reality, the maximum applied stress seems to generate at the center of loading platens, and decreases as the distance from the center increases. Eventually, the applied load seems to disappear at the edges of the contact area. In order to satisfy this boundary condition on the contact area, the cosine curve was adopted as the distribution of loading applied on loading platens in a theoretical model for diametrical loading test by authors. The distribution of tensile stress on the loading radius under this loading is similar to that under uniform loading[10]. However, maximum value of displacement occurring at the center of the loading platens does not agree with the results from experiments [11]. Furthermore, the hybrid model that consists with uniform load and the cosine curved load is applied for the distribution of stress on the contact area [12]. However, the ratio of magnitude of the cosine curved loading to that of uniform loading was not estimated correctly in this model.

In this study, the ratio of magnitude of cosine curved loading to that of the uniform loading is re-examined. The boundary condition proposed in this study is fixed into the orthotropic stress function by Lekhnitskii [13], the tensile stress generating along loading radius is obtained for selected orthotropic ratio and direction. Furthermore, the results in this study are compared with those from previous works.

\section{Fundamental Equations}

In this paper, the problem of the application of opposite concentrated loads $P$ to the diametrical axis of an orthotropic column specimen of radius $a$ is treated as shown in Fig. 1; 
Here, $\varphi$ is the angle between the loading axis and the principal elastic axis, and $E_{1}$ and $E_{2}$ are the respective elastic moduli in the direction of the principal elastic axis.

By denoting the loading axis as $x$, and the perpendicular direction in the plane of the loading axis as $y$, the following equations representing the resultant forces on surface $X_{n}$ and $Y_{n}$, going anticlockwise from the $x$-axis in the $x$ and $y$ directions, on the boundary circumference of a column-shaped specimen, which are related to stress components $\sigma_{x}, \sigma_{y}$ and $\tau_{x y}$ are obtained:

$$
\left.\begin{array}{l}
X_{n}=\sigma_{x} \cos (n, x)+\tau_{x y} \cos (n, y), \\
Y_{n}=\tau_{x y} \cos (n, x)+\sigma_{y} \cos (n, y) .
\end{array}\right\}
$$

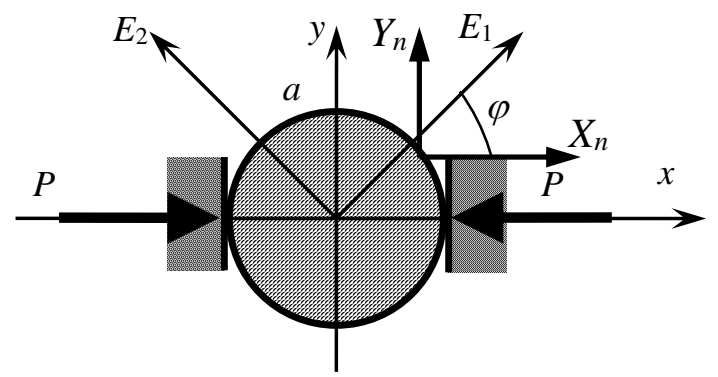

Fig. 1. Orthotropic circular disk under diametrical compression

The stress components $\sigma_{x}, \sigma_{y}$ and $\tau_{x y}$, and the displacement components $u_{x}$ and $u_{y}$ are expressed by the following[13].

$$
\begin{aligned}
& \sigma_{x}=2 \operatorname{Re}\left[\mu_{1}^{2} \Phi_{1}^{\prime}\left(z_{1}\right)+\mu_{2}^{2} \Phi_{2}^{\prime}\left(z_{2}\right)\right], \\
& \sigma_{y}=2 \operatorname{Re}\left[\Phi_{1}^{\prime}\left(z_{1}\right)+\Phi_{2}^{\prime}\left(z_{2}\right)\right] \text {, } \\
& \tau_{x y}=-2 \operatorname{Re}\left[\mu_{1} \Phi_{1}^{\prime}\left(z_{1}\right)+\mu_{2} \Phi_{2}{ }^{\prime}\left(z_{2}\right)\right] . \\
& \left.u_{x}=2 \operatorname{Re}\left[p_{1} \Phi_{1}\left(z_{1}\right)+p_{2} \Phi_{2}\left(z_{2}\right)\right]\right), \\
& \left.u_{y}=2 \operatorname{Re}\left[q_{1} \Phi_{1}\left(z_{1}\right)+q_{2} \Phi_{2}\left(z_{2}\right)\right]\right\}
\end{aligned}
$$

Here, Re denotes the real part of the complex functions in brackets. ' means the first derivative of $\Phi_{k}\left(z_{k}\right)$ with respect to $z_{k}$.

The integral calculi about resultant forces for the $x$-directions and $y$-directions on the surface are expanded as Fourier series with $M$ terms, as follows:

$$
\left.\begin{array}{r}
\int_{0}^{s} X_{n} d s=\beta_{0}+\sum_{m=1}^{M}\left(\beta_{m} e^{i m \theta}+\bar{\beta}_{m} e^{-i m \theta}\right), \\
-\int_{0}^{s} Y_{n} d s=\alpha_{0}+\sum_{m=1}^{M}\left(\alpha_{m} e^{i m \theta}+\bar{\alpha}_{m} e^{-i m \theta}\right),
\end{array}\right\}
$$

Here, the bar denotes the complex conjugate. $\alpha_{m}$ and $\beta_{m}$ for the problem in this paper are determined in the next section.

The complex stress functions for an orthotropic circular plate with radius $a$ are expanded as the series expressed below[13]: 


$$
\left.\begin{array}{l}
\Phi_{1}\left(z_{1}\right)=A_{0}+A_{1} z_{1}+\sum_{m=2}^{M} A_{m} P_{1 m}\left(z_{1}\right), \\
\Phi_{2}\left(z_{2}\right)=B_{0}+B_{1} z_{2}+\sum_{m=2}^{M} B_{m} P_{2 m}\left(z_{2}\right) .
\end{array}\right\}
$$

Here $P_{k m}\left(z_{k}\right)(k=1,2)$ is a power series of the $m$-th order anis expressed by the following equation.

$$
\begin{aligned}
P_{k m}\left(z_{k}\right)= & -\frac{1}{a^{m}\left(1-i \mu_{k}\right)^{m}} \\
& \left.\times\left\{\left(z_{k}+\sqrt{z_{k}^{2}-a^{2}\left(1-\mu_{k}^{2}\right.}\right)\right)^{2}+\left(z_{k}-\sqrt{z_{k}^{2}-a^{2}\left(1-\mu_{k}^{2}\right.}\right)^{2}\right\} \quad(k=1,2) .
\end{aligned}
$$

where, $\mu_{k}$ are the roots of the characteristic equation shown below.

$$
a_{11} \mu^{4}-2 a_{16} \mu^{3}+\left(2 a_{12}+a_{66}\right) \mu^{2}-2 a_{26} \mu+a_{22}=0 \text {. }
$$

$a_{i j}(i, j=1,2,6)$ represents elastic compliances for plane stress condition. The complex variable $z_{k}$ and power series $P_{k m}\left(z_{k}\right)$ are represented on the cylindrical boundary as follows:

$$
\left.\begin{array}{l}
z_{k}=\frac{a}{2}\left\{\left(1-\mu_{k}\right) e^{i \theta}+\left(1+i \mu_{k}\right) e^{-i \theta}\right\}, \\
P_{k m}=-\left(e^{i m \theta}+t_{k}^{m} e^{-i m \theta}\right), \quad t_{k}=\frac{1+i \mu_{k}}{1-i \mu_{k}} . \quad(k=1,2)
\end{array}\right\}
$$

By using the complex functions $\Phi_{k}\left(z_{k}\right)$, the integral calculi on resultant stresses are expressed as follows:

$$
\left.\begin{array}{l}
\int_{0}^{s} X_{n} d s=\operatorname{Re}\left[\mu_{1} \Phi_{1}\left(z_{1}\right)+\mu_{2} \Phi_{2}\left(z_{2}\right)\right], \\
-\int_{0}^{s} Y_{n} d s=\operatorname{Re}\left[\Phi_{1}\left(z_{1}\right)+\Phi_{2}\left(z_{2}\right)\right] .
\end{array}\right\}
$$

By substituting Eq. (8) into Eq. (4), and obtained equations into Eq. (9), the equations for assigning the resultant stress on the boundary are obtained. Furthermore, the coefficients of the corresponding terms of $e^{i m \theta}$ and $e^{-i m \theta}(1 \leq m \leq M)$ are compared with each other, resulting in the following equations:

$$
\left.\begin{array}{c}
A_{1}+B_{1}+\bar{A}_{1}+\quad \bar{B}_{1}=\frac{1}{a}\left(\alpha_{1}+\bar{\alpha}_{1}\right), \\
\mu_{1} A_{1}+\mu_{2} B_{1}+\bar{\mu}_{1} \bar{A}_{1}+\bar{\mu}_{2} \bar{B}_{1}=\frac{1}{i b}\left(\bar{\alpha}_{1}-\alpha_{1}\right)=\frac{1}{a}\left(\beta_{1}+\bar{\beta}_{1}\right), \\
\mu_{1}^{2} A_{1}+\mu_{2}^{2} B_{1}+\bar{\mu}_{1}^{2} \bar{A}_{1}+\bar{\mu}_{2}^{2} \bar{B}_{1}=\frac{1}{i b}\left(\bar{\beta}_{1}-\beta_{1}\right) .
\end{array}\right\}
$$




$$
\begin{aligned}
& A_{m}+\quad B_{m}+\bar{t}_{1}^{m} \bar{A}_{m}+\bar{t}_{2}^{m} \bar{B}_{m}=-\alpha_{m}, \\
& \mu_{1} A_{m}+\mu_{2} B_{m}+\bar{\mu}_{1} \bar{t}_{1}^{m} \bar{A}_{m}+\bar{\mu}_{2} \bar{t}_{2}^{m} \bar{B}_{m}=-\beta_{m}, \\
& t_{1}{ }^{m} A_{m}+t_{2}{ }^{m} B_{m}+\bar{A}_{m}+\quad \bar{B}_{m}=-\bar{\alpha}_{m}, \\
& \mu_{1} t_{1}{ }^{m} A_{m}+\mu_{2} t_{2}{ }^{m} B_{m}+\bar{\mu}_{1} \bar{A}_{m}+\bar{\mu}_{2} \bar{B}_{m}=-\bar{\beta}_{m} \text {. } \\
& (m \geq 2)
\end{aligned}
$$

For the case of $m=1$, there are only three equations for the four unknown quantities of the real and imaginary parts of $A_{1}$ and $B_{1}$, respectively, so these equations are unsolved. For this reason, the condition that the rigid body rotation is zero is additionally introduced, resulting in the following equation[14].

$$
\left(q_{1}-\mu_{1} p_{1}\right) A_{1}+\left(q_{2}-\mu_{2} p_{2}\right) B_{1}+\left(\bar{q}_{1}-\bar{\mu}_{1} \bar{p}_{1}\right) \bar{A}_{1}+\left(\bar{q}_{2}-\bar{\mu}_{2} \bar{p}_{2}\right) \bar{B}_{1}=0 .
$$

where

$$
\left.\begin{array}{ll}
p_{1}=a_{11} \mu_{1}^{2}+a_{12}, & p_{2}=a_{11} \mu_{2}^{2}+a_{12}, \\
q_{1}=\left(a_{12} \mu_{1}^{2}+a_{22}\right) / \mu_{1}, & q_{2}=\left(a_{12} \mu_{2}^{2}+a_{22}\right) / \mu_{2} .
\end{array}\right\}
$$

By adding Eq. (12) to Eq. (10), $A_{1}$ and $B_{1}$ can be obtained completely.

\section{Equations and mathematics}

In this paper, the shape which consists with uniform loading at the center and cosine curved loading at both edges is proposed as distribution of loading on the contact area $\omega / 2$. As shown in Fig. 2, from center of contact area, the uniform loading whose width is $\omega / 8$ are applied on both side. While, from both edge of uniform loading, cosine shaped load whose width is $\omega / 8$ are applied on both side and loading is disappeared on the edge of contact area. Namely, this means that $50 \%$ of contact area is covered with uniform loading.

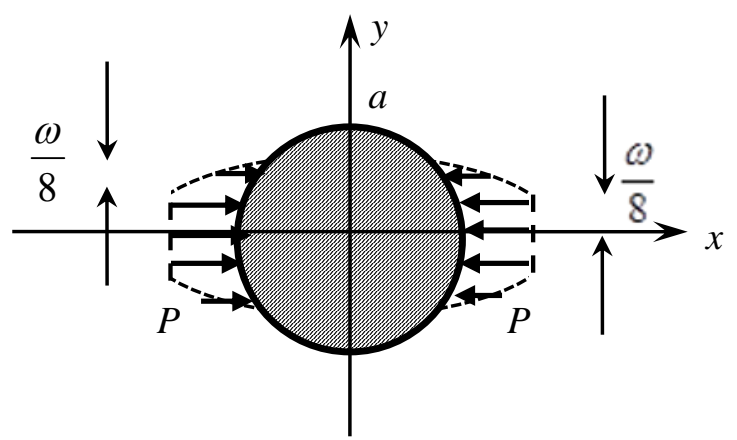

Fig. 2. Boundary condition in this study

Fourier coefficients in Eq. (4) are expressed as shown in Eq. (14) for this problem.

$$
\alpha_{m}=0, \quad \beta_{m}=-i b_{m} \text {. }
$$

$b_{m}$ are able to be obtained through Fourier integral. The first equation in Eq. (4) is an odd function in this problem. Therefore, Fourier sine transform is able to be adopted for this problem. Therefore, the first equation in Eq. (4) is able to be treated as the function between 0 and $\pi$ because it is the odd function. 
Let us define the ratio of magnitude of uniform load to that of the cosine curved loading. Fig. 3 shows the magnitude of both load on the half contact area. The magnitudes of uniform loading and cosine curved loading are shown as areas in this figure, $A_{l 1}$ and $A_{l 2}$, respectively. Because contact areas of both loading are same as $\omega / 8$, the ratio of $A_{l 1}$ to $A_{l 2}$ is shown in Eq. (15)

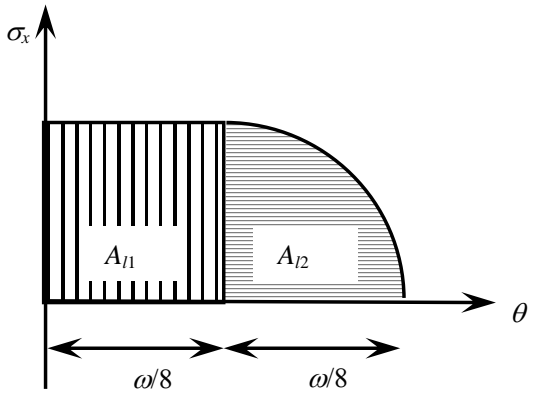

$$
A_{l 1}: A_{l 2}=1: \frac{2}{\pi}
$$

Fig. 3. Magnitude of stress on the loading area

Therefore, the distribution of resultant force in $x$ direction, obtained by integration of $\sigma_{x}$ with $\theta$ in Fig. 3, is shown in Fig. 4, where $A_{l a}=A_{l 1}+A_{l 2}$.

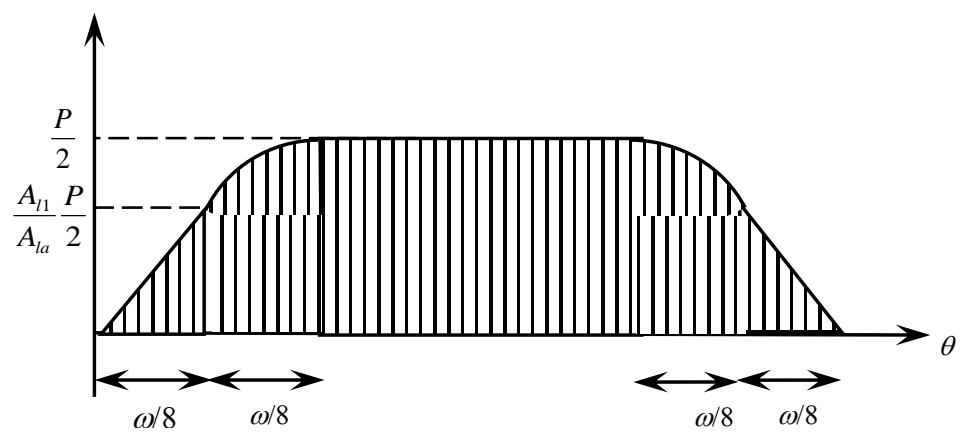

Fig. 4. Distribution of resultant force

The distribution of resultant force in Fig. 4 is able to be formulated into Eq. (16); 


$$
\int X_{n} d s= \begin{cases}\frac{P}{2} \frac{A_{l 1} / A_{l a}}{\omega / 8} \theta, & \left(0 \leq \theta<\frac{\omega}{8}\right) \\ \frac{P}{2} \frac{A_{l 1}}{A_{l a}}-\frac{P}{2} \frac{A_{l 2}}{A_{l a}} \cos \frac{\pi / 2}{\omega / 8} \theta, & \left(\frac{\omega}{8} \leq \theta<\frac{\omega}{4}\right) \\ \frac{P}{2}, & \left(\frac{\omega}{4} \leq \theta<\pi-\frac{\pi}{4}\right) \\ \frac{1}{4}+C_{1} \sin \frac{\pi / 2}{\omega / 8} \theta+C_{2} \cos \frac{\pi / 2}{\omega / 8} \theta, & \left(\pi-\frac{\omega}{4} \leq \theta<\pi-\frac{\omega}{8}\right) \\ \frac{1}{2} \frac{A_{l 11} / A_{l a}}{\omega / 8}(\pi-\theta) . & \left(\pi-\frac{\omega}{8} \leq \theta<\pi\right)\end{cases}
$$

Where,

$$
C_{1}=-\frac{1}{4} \frac{A_{l 2}}{A_{l a}} \cos \frac{4 \pi}{\omega}\left(\pi-\frac{\omega}{8}\right), \quad C_{2}=\frac{1}{4} \frac{A_{l 2}}{A_{l a}} \sin \frac{4 \pi}{\omega}\left(\pi-\frac{\omega}{8}\right) .
$$

As mentioned before, the distribution of resaltant force is represented using the odd function. Therefore, Fourier coefficients for Eq. (16) is obtained as shown below.

$$
\begin{aligned}
b_{m}=\frac{2 P}{\pi}[ & \int_{0}^{\omega / 8} \frac{4}{\omega} \frac{A_{l 1}}{A_{l a}} \sin m \theta d \theta+\int_{\omega / 8}^{\omega / 4} \frac{1}{2 A_{l a}}\left\{A_{l 1}-A_{l 2} \cos \frac{4 \pi}{\omega} \theta\right\} \sin m \theta d \theta \\
& +\frac{1}{2} \int_{\omega / 4}^{\pi-\omega / 4} \sin m \theta d \theta \\
& +\int_{\pi-\omega / 4}^{\pi-\omega / 8}\left\{\frac{1}{4}+C_{1} \sin \frac{4 \pi}{\omega} \theta+C_{2} \cos \frac{4 \pi}{\omega} \theta\right\} \sin m \theta d \theta \\
& \left.+\int_{\pi-\omega / 8}^{\pi} \frac{4}{\omega} \frac{A_{l 1}}{A_{l a}}(\pi-\theta) \sin m \theta d \theta\right] .
\end{aligned}
$$

The right side of Eq. (18) is calculated into Eq. (19).

$$
\begin{aligned}
b_{m}=\frac{2}{\pi} & \left(B_{1 m}^{*}+B_{2 f m}^{*}+B_{2 b 1 m}^{*}+B_{2 b 2 m}^{*}+B_{3 m}^{*}\right. \\
& \left.+B_{4 f m}^{*}+B_{4 c 1 m}^{*}+B_{4 c 2 m}^{*}+B_{4 b 1 m}^{*}+B_{4 b 2 m}^{*}+B_{5 f m}^{*+}+B_{5 b m}^{*}\right) .
\end{aligned}
$$

where 


$$
\left.\begin{array}{rl}
B_{1 m}^{*}= & -R_{1}\left(\frac{1}{2 m} \cos \frac{m \omega}{8}-\frac{4}{m^{2} \omega} \sin \frac{m \omega}{8}\right), \\
B_{2 f m}^{*}= & \frac{R_{1}}{m} \sin \frac{3 m \omega}{16} \sin \frac{m \omega}{16}, \\
B_{2 b 1 m}^{*}=\frac{R_{2}}{4} D_{p m}\left(\cos \frac{\omega \theta_{1 p m}}{4}-\cos \frac{\omega \theta_{1 p m}}{8}\right), \\
B_{2 b 2 m}^{*}=\frac{R_{2}}{4} D_{n m}\left(\cos \frac{\omega \theta_{1 n m}}{4}-\cos \frac{\omega \theta_{1 n m}}{8}\right), \\
B_{3 m}^{*}=\frac{1}{2 m}\left\{\cos \frac{m \omega}{4}-\cos m \theta_{4}\right\}, \\
B_{4 f m}^{*}=\frac{1}{4 m}\left\{\cos m \theta_{4}-\cos m \theta_{8}\right\}, \\
B_{4 c 1 m}^{*}=-\frac{C_{1}}{2} D_{p m}\left\{\sin \theta_{1 p m} \theta_{8}-\sin \theta_{1 p m} \theta_{4}\right\}, \\
B_{4 c 2 m}^{*}=-\frac{C_{1}}{2} D_{n m}\left\{\sin \theta_{1 n m} \theta_{8}-\sin \theta_{1 n m} \theta_{4}\right\}, \\
B_{4 b 1 m}^{*}=-\frac{C_{2}}{2} D_{p m}\left\{\sin \theta_{1 p m} \theta_{8}-\sin \theta_{1 p m} \theta_{4}\right\}, \\
B_{4 b 2 m}^{*}=-\frac{C_{2}}{2} D_{n m}\left\{\sin \theta_{1 n m} \theta_{8}-\sin \theta_{1 n m} \theta_{4}\right\}, \\
B_{5 f m}^{*}=\frac{4 R_{1}}{m \omega}\left\{(-1)^{m+1}+\cos m \theta_{8}\right\}, \\
B_{5 b m}^{*}=-\frac{4 R_{1}}{m \omega \pi}\left\{\pi(-1)^{n+1}+\theta_{8} \cos m \theta_{8 m}-\frac{1}{m} \sin m \theta_{8}\right\} . \\
\theta_{1 p m}=\frac{A_{l 1}}{A_{l a}}, R_{2}=\frac{A_{l 2}}{A_{l a}}, D_{p m}=\frac{4 \pi}{\omega}, \theta_{1 n m}=m-\frac{\omega \pi}{\omega}, \theta_{4}=\pi-\frac{\omega}{4}, \theta_{8}=\pi-\frac{\omega}{8} .
\end{array}\right\}
$$

\section{Results and Discussion}

Discussion about the convergence and accuracy for values from calculation in this study is dealt in another work by authors. Futhermore, fracture happens under contact area from 4.4 to $5.1 \%$ in Tage stone and $6.4 \%$ in cement modified soil [15]. Therefore, the distribution of tensile stress in specimens under contact area $4 \%$ and $8 \%$ is dealt in this paper.

Fig. 5 shows the distribution of tensile stress on the loading radius in isotropic specimen $\left(E_{2} / E_{1}=0.98\right)$ from present result, under uniform loading, cosign shaped loading and concentrated force. The longitudinal axis represents the normal stress to loading direction. This stress is normalized by the tensile strength for isotropic disk $P /(\pi a)$ shown in the specifications [16]. The transverse axis represents the distance from center of the disk. This 
distance is also normalized by length of specimen's radius $a$. The uniform distribution of tensile stress appears under concentrated force that is used as the standard of measurement for tensile strength[16]. On the other hand, the maximum value of tensile stress appears at the center of specimen at first, the value of tensile stress decreases gently as the distance from center of specimen $x / a$ increases secondly, decreases suddenly near the loading platen at last under all loadings except concentrated force. The distribution of tensile stress from present study seems to be almost same as those from uniform loading or cosign shaped loading in contact area $4 \%$. In contact area $8 \%$, the tensile stress starts to decrease at the point where $x / a$ is about 0.5 under present result, while about 0.3 under cosign shaped loading and about 0.2 under uniform loading.

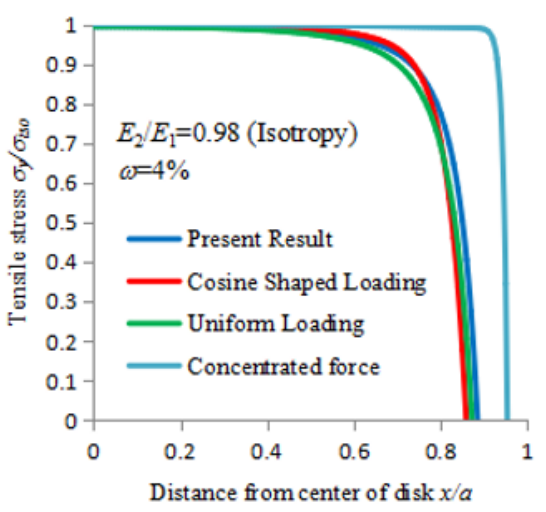

(a) Contct area $4 \%$

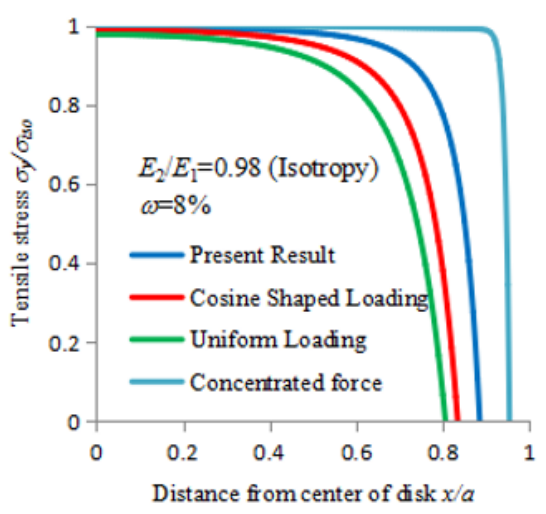

(b) Contact area $8 \%$

Fig.5 Tensile stress on the loading radius in isotropic specimen

Comparisons of the distribution of tensile stress on the loading radius among sevral loadings are also carried out for orthotropic specimen $\left(E_{2} / E_{1}=1.5\right)$. Fig. 6 shows when $\varphi=0$, namely the loading is carried out in softer axis. Fig. 7 shows when $\varphi=\pi / 2$, namely the loading is carried out in harder axis. Fig. 8 shows when $\varphi=\pi / 4$, namely the loading is carried out in the intermediate direction between harder and softer axes. When the loading direction is in principal elastic axis (i. e. $\varphi=0, \pi / 2$ ), the obvious maximum value appears near the loading platen under concentrated force. While the maximum value appears at the distance from center of specimen $x / a$ is about 0.7 under present result, uniform loading and cosign curved loading when the contact area is $4 \%$. Furthermore, uniform distribution appears between $x / a=0$ and about 0.6 , and the maximum value doesn't appear when the contact area is $8 \%$. In these figures, the value of tensile stress generating near the loading platen under concentrated force is largest, and distributions of tensile stress under present result, uniform loading and cosign curved loading are almost same. As shown in authors' another work, the obvious maximum value of displacement generate under concentrated force and this displacement is different from the actual behavior in diametrical loading test using flat loading platen. Therefore, it seems that the distributions of tensile stress obtained under distributed loading on loading platen are more reliable, especially near the loading platen than that under concentrated force.

When the loading is carried out in softer axis $(\varphi=0)$, the value of maximum tensile stress is about 1.0 for $4 \%$ of contact area and about 0.95 for $8 \%$. Therefore, the method to obtain the tensile strength for isotropic specimen is available in this loading direction and orthotropy ratio. When the loading is carried out in harder axis $(\varphi=\pi / 2)$, the tensile stress generating on the loading radius is smaller than that in softer axis because the deformation under loading in harder axis is smaller than that in softer axis. The value of maximum tensile stress is 
about 0.82 for $4 \%$ of contact area and about 0.76 for $8 \%$. Therefore, the tensile strength is estimated about $80 \%$ to the value obtained from the method for isotropic specimen.

When the loading is carried out in the intermediate direction between harder and softer axis $(\varphi=\pi / 2)$, the maximum value of tensile stress generates at the center of specimen (i. e. $x / a=0)$ under all shapes of loading distribution. Therefore, the tensile strength is estimated about $120 \%$ for the value obtained from the method for isotropic specimen.

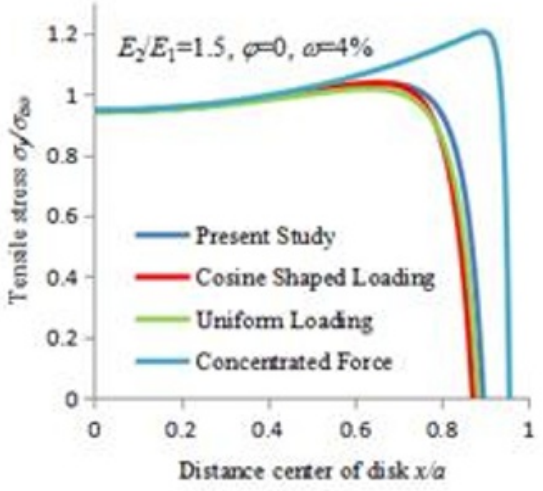

(a) Contct area $4 \%$

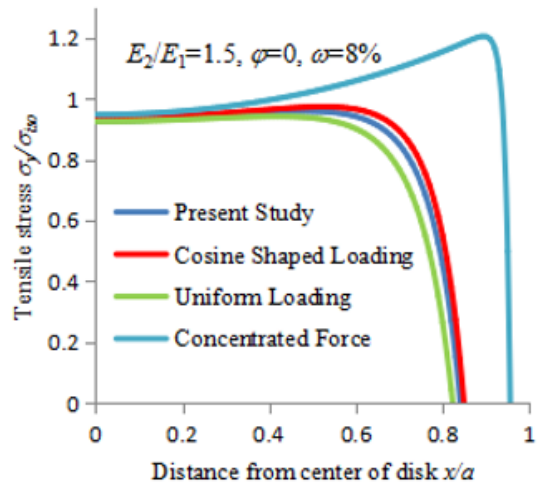

(b) Contact area $8 \%$

Fig. 6 . Tensile stress on the loading radius in orthotropic specimen $\left(E_{2} / E_{1}=1.5, \varphi=0\right)$

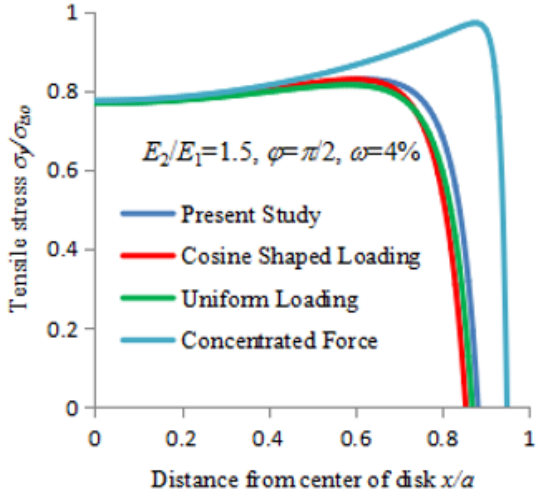

(a) Contct area $4 \%$

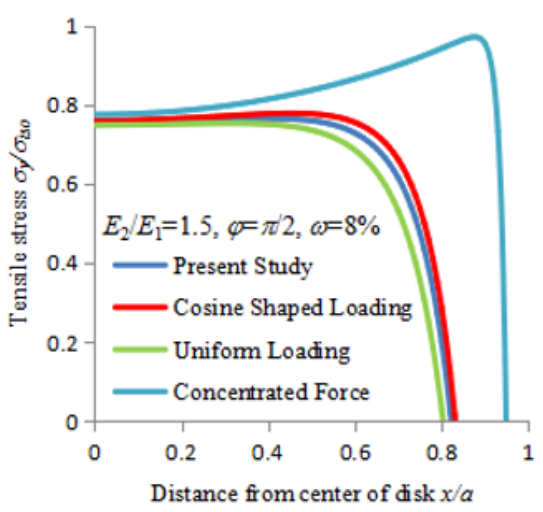

(b) Contact area $8 \%$

Fig. 7. Tensile stress on the loading radius in orthotropic specimen $\left(E_{2} / E_{1}=1.5, \varphi=\pi / 2\right)$ 


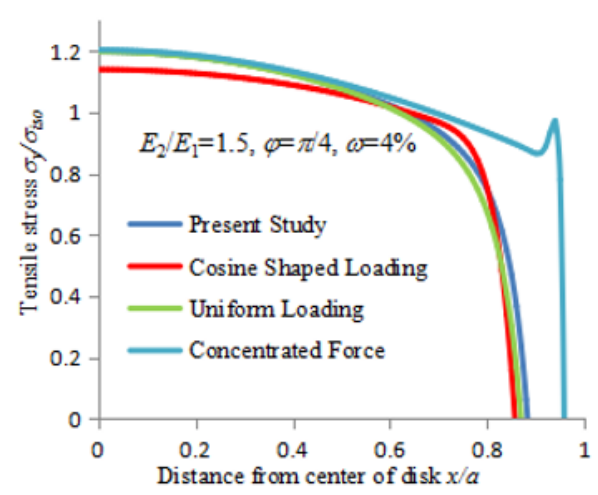

(a) Contct area $4 \%$

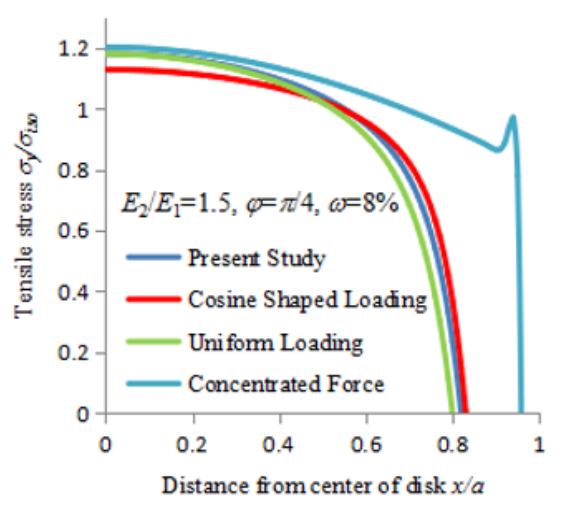

(b) Contact area $8 \%$

Fig. 8. Tensile stress on the loading radius in orthotropic specimen $\left(E_{2} / E_{1}=1.5, \varphi=\pi / 4\right)$

\section{Conclusion}

Previous studies either used point, uniform or cosine shaped loading as stress distribution on the loading platen for theoretical models in diametrical loading test. The present study adopts combination of loading consists of cosine shape and uniformly distributed load to satisfy the boundary conditions at the contact area between specimen and loading platens. The distributions of tensile stress along loading radius are calculated for selected orthotropy ratio, directions and contact areas, and compared with some results from previous studies. It is shown that the value of tensile stress generating near the loading platen in the result from present study, that is almost same as that from uniform loading, is smaller than that from concentrated force. Furthermore, the maximum value of tensile stress in orthotropic $\left(E_{2} / E_{1}=1.5\right)$ specimen from present study is almost same as that in isotropic specimen when loading is carried out in softer axis, and smaller than that when in harder axis and larger than that in intermediate direction between softer and harder axis.

\section{References}

1. I. S. Sokolnikoff, Mathematical theory of elasticity, McGraw-Hill, 476 (1956).

2. F. V. Cauweleart, and B. Eckmann, Indirect tensile test applied to anisotropic materials, Materials and structures, 27, 54 (1994).

3. J. Claesson, and B. Bohloli, Brazilian test: stress field and tensile strength of anisotropic rocks using an analytical solution, Int. J. of Rock Mech. and Mining Sci., 39, 991 (2002).

4. G. E. Exadaktylos, On the constraints and relations of elastic constants of transversely isotropic geomaterials, Int. J. of Rock Mech. and Mining Sci., 38, 941 (2001).

5. G. E. Exadaktylos, and K.N. Kaklis, Applications of an explicit solution for the transversely isotropic circular disc compressed diametrically, Int. J. of Rock Mech. and Mining Sci., 38, 227 (2001).

6. C. S. Chen, , E. Pan and B. Amadei, Determination of Deformability and Tensile Strength of Anisotropic Rock Using Brazilian Tests, Int. J. of Rock Mech. and Mining Sci., 35, 43 (1998). 
7. Ch. F. Markides, D. N. Pazis, and S. K. Kourkoulis, Closed full-fill solutions for stress and displacements in the Brazilian disk under distributed radial load, Int. J. of Rock Mech. and Mining Sci., 47, 227 (2010).

8. R. K. Lemmon and D. M. Blackketter, Stress Analysis of an Orthotropic Material under Diametral Compression, Experimental Mech. 36, 204 (1996).

9. T. Tsutsumi, and K. Hirashima, Analysis of Orthotropic Circular Disks and Rings under Diametrical Loading, Structural Eng. and Mech., 9(1), 37 (2000).

10. T. Tsutsumi, and S. Kukino, Distribution of Tensile Stress under Modified Boundary Condition in Theoretical Solution for Diametrical Compression Test, Proc. 13th ISRM Congress, CD-ROM (2015).

11. S. Kukino, and T. Tsutsumi, Modification of boundary condition in theoretical model for diametrical compression test, Proc. 4th International Symposium on Technology for Sustainability, CD-ROM (2014).

12. T. Tsutsumi, R. A. Abdullah, and M. F. M. Amin, Theoretical model using two kinds of function for distribution of applied load in Brazilian Test, Proc. 9th Asian Rock Mechanics Symposium, USB-Flash memory (2016).

13. S. G. Lekhnitskii, Anisotropic Plate, 141, Gordon \& Breach (1968).

14. S. Kawakubo, T. Tsutsumi, and K. Hirashima, Stress and Displacement Fields for an Anisotropic Elliptical Disk Subjected to Arbitrary Loads at Boundary (in Japanese), Trans. JSME Series A, 62, 1626 (1996).

15. Y. Aono, K. Tani, T. Okada, and M. Sakai, The mechanism of failure near the loading point in the splitting tensile strength test on Tage Stone (in Japanese), Proc. of 41th Rock Mech. Symposium in Japan, 157 (2012).

16. R. Ulusay and J. A. Hudson, The complete ISRM suggested methods for rock characterization, testing and monitoring, 1974-2006, 181 (2006). 